\title{
Altered expression of DLG1-AS1 distinguished papillary thyroid carcinoma from benign thyroid nodules
}

\author{
Tao He${ }^{1}$, Huan Wang ${ }^{1}$, Jiangming Sun ${ }^{1}$, Jie Wu${ }^{1}$, Fakuo Gong ${ }^{1}$, Shujun $\mathrm{Li}^{1}$, Hui Wang ${ }^{1}$ and Yufeng $\mathrm{Li}^{2^{*}}$ (D)
}

\begin{abstract}
Background: Benign thyroid nodules (BTN) are frequently diagnosed as papillary thyroid carcinoma (PTC), leading to unnecessary treatment. We found that plasma IncRNA DLG1-AS1 was upregulated in PTC patients but not in BTN patients and healthy controls.

Methods: In this study DLG1-AS1 and miR-199a-3p in plasma of both PTC patients and BTN patients were detected by $\mathrm{qPCR}$. ROC curve analysis was performed for diagnostic analysis. Overexpression experiments were performed to analyze the interaction between DLG1-AS1 and miR-199a-3p. CCK-8 assay was performed to analyze cell proliferation.

Results: In this study, upregulation of DLG1-AS1 distinguished PTC patients from BTN patients and healthy controls. Plasma miR-199a-3p was downregulated in PTC patients compared with healthy controls and BTN patients. Plasma levels of miR-199a-3p were inversely correlated in PTC patients, but not in BTN patients and healthy controls. miR199a-3p overexpression failed to significantly affect DLG1-AS1, while DLG1-AS1 overexpression resulted in downregulated miR-199a-3p, In addition, DLG1-AS1 overexpression promoted the proliferation of PTC cells. miR199a-3p overexpression played an opposite role and attenuated the effects of DLG1-AS1 overexpression.
\end{abstract}

Conclusions: Therefore, DLG1-AS1 may promote PTC by downregulating miR-199a-3p.

Keywords: DLG1-AS1, Papillary thyroid carcinoma, Benign thyroid nodules, miR-199a-3p, Proliferation

\section{Background}

In 2018 , thyroid cancer affected 130,889 new cases in males and 436,344 new cases in females, and the incidence rate in females is more than 3 times higher than in males [1]. Papillary thyroid carcinoma (PTC) is the most common type of thyroid cancer [2]. PTC is a type of slow-growing, well-differentiated, and localized thyroid cancer, although it can metastasize [2]. Comparing to other malignancies mortality rate of thyroid cancer is relatively low, which is mainly due to its less aggressive nature and slow development [3]. In 2018, thyroid cancer only caused 41,000 deaths, which is only about $7 \%$ of the newly diagnosed cases [1]. However, metastasis will inevitably occur in some cases of PTC, leading to poor

\footnotetext{
* Correspondence: suemwvjlds99@163.com

2Department of Ultrasound, Panzhihua Central Hospital, No. 34 YiKang street, Eastern DistrictSichuan Province 617067 Panzhihua City, People's Republic of China

Full list of author information is available at the end of the article
}

prognosis $[4,5]$. Therefore, early diagnosis is still critical. However, PTC in some cases can be misdiagnosed as other thyroid disorders, such as benign thyroid nodules [6]. Therefore, how to distinguish PTC from BTN is a challenge in clinical treatment.

Taking advantage of the non-invasive nature, circulating markers have been widely used for disease prediction [7]. Long no-coding RNAs ( $>200 \mathrm{nt}$, lncRNAs) are RNA transcripts encoding no protein [8]. However, lncRNAs can regulate the expression of protein-coding genes at multiple levels to participate in diverse pathological and physiological processes [8]. Although lncRNAs are usually spatially and/or temporally expressed, they can be released into blood circulation system after synthesis to traffic to other positions of the body $[9,10]$. Therefore, the dysregulation of lncRNAs under pathological conditions may be reflected by its levels in plasma $[9,10]$. DLG1-AS1 is a recently identified oncogenic lncRNA in

(c) The Author(s). 2019 Open Access This article is distributed under the terms of the Creative Commons Attribution 4.0 International License (http://creativecommons.org/licenses/by/4.0/), which permits unrestricted use, distribution, and reproduction in any medium, provided you give appropriate credit to the original author(s) and the source, provide a link to the Creative Commons license, and indicate if changes were made. The Creative Commons Public Domain Dedication waiver (http://creativecommons.org/publicdomain/zero/1.0/) applies to the data made available in this article, unless otherwise stated. 
cervical cancer [11]. MiR-199a-3p is a well-characterized tumor suppressive miRNA in cancer biology [12-14]. We observed the inverse correlation between DLG1-AS1 and miR-199a-3p from our preliminary bioinformatics analysis. This study aimed to explore the roles of DLG1AS1 and miR-199a-3p in PTC and analyze the clinical values of DLG1-AS1 for this disease.

\section{Methods}

\section{Research subjects}

Research subjects in this study included 60 PTC patients (16 males and 44 females; age: 29 to 67 years; mean: $48.1 \pm$ 5.5 years), 60 BTN patients (16 males and 44 females; age: 30 to 67 years; mean: $48.3 \pm 5.9$ years), and 60 healthy controls (16 males and 44 females, age: 28 to 67 years; mean: $47.9 \pm 5.0$ years). This study passed the review board of Panzhihua Central Hospital. All the participants were selected in Panzhihua Central Hospital during April 2015 and April 2019. PTC and BTN patients were confirmed by histopathological biopsy. Patients' inclusion criteria: 1) newly diagnosed cases; 2 ) no therapies were initiated before the admission of patients. Exclusion criteria: 1) recurrent cases; 2) other clinical disorders were observed; 3) history of malignancy; 4) therapies were performed. The 60 PTC patients were at AJCC stage I $(n=28)$ and II $(n=32)$. All participants were educated the principle of experimental designed and informed consent was signed by all of them.

\section{Blood extraction and plasma preparation}

On the day of admission (before the initiation of therapies), fasting blood $(5 \mathrm{ml})$ was extracted from all participants. To separate plasma, blood was injected into EDTA tubes and the tubes were centrifuged for $20 \mathrm{~min}$ at $1200 \mathrm{~g}$.

\section{PTC cells and transient transfections}

IHH-4 and MDA-T32 (ATCC, USA) two human PTC cell lines were included. The cell culture medium for both cell lines was prepared by mixing DMEM and FBS with a ratio of 9:1. Cell culture conditions were 95\%, $37^{\circ} \mathrm{C}$, and $5 \% \mathrm{CO}_{2}$. DLG1-AS1 expression vector was constructed using pcDNA3.1 vector (GenePharma, Shanghai, China). Scramble microRNA mimics were designed as negative control microRNA (NC) and miR199a-3p were both from RIBOBIO77 (Guangzhou, China). At confluence of 70-80, IHH-4 and MDA-T32 cells were harvested and counted, followed by the transfection of $40 \mathrm{nM}$ miRNA (NC miRNA as NC group) or $10 \mathrm{nM}$ vector (empty vector as $\mathrm{NC}$ group) into $3 \times 10^{6}$ cells through transient transfections mediated by lipofectamine 2000 (Invitrogen, USA). Control (C) cells for all groups were untransfected cells. All subsequent experiments were performed using cells harvested at $24 \mathrm{~h}$ post-transfection.

\section{RNA extraction}

Total RNAs in $0.2 \mathrm{ml}$ plasma and $3 \times 10^{5}$ cells were extracted using Trizol reagent (Invitrogen, USA). To harvest miRNAs, RNA samples were precipitated and washed using $85 \%$ ethanol. All RNA samples were digested with DNase I for $2 \mathrm{~h}$ to remove genomic DNA.

\section{QPCR}

AMV reverse transcriptase (GIBCO, USA) was used to transcribe total RNAs into cDNA. Using cDNA as template, FastStart Universal SYBR Green Master (Sigma-Aldrich) was used to prepare qPCR mixtures to measure the expression levels of DLG1-AS1. To measure the expression levels of miR-199a-3p, All-in-One ${ }^{\mathrm{Tu}}$ miRNA qRTPCR Reagent Kit (Genecopoeia) was used to perform all steps, including $1^{\prime}$ polyadenylation, reverse transcription and qPCR assays (U6 as endogenous control). $2^{-\Delta \Delta C T}$ method was used for data normalizations and three replicates were included in each experiment.

\section{Cell proliferation assay}

IHH-4 and MDA-T32 cells were harvested at $24 \mathrm{~h}$ posttransfection. Cells were counted and $1 \mathrm{ml}$ DMEM (10\% FBS) was used to resuspend cell pellets $\left(3 \times 10^{4}\right.$ cells $)$ to make single cell suspensions. A 96-well cell plate was used to cultivate cells $(0.1 \mathrm{ml}$ per well $)$ under the aforementioned conditions. CCK- 8 solution (10 $\mu$ l, SigmaAldrich) was added into each well at $4 \mathrm{~h}$ before the end of cell culture. After cell culture was terminated, $10 \mu \mathrm{l}$ DMSO was added into each well and OD values at 450 nm were measured.

\section{Statistical analysis}

Experiments were performed in 3 biological replicates and data were expressed as mean values. ANOVA (oneway) in combination with Tukey test were used to explore differences among different groups of participants or among different cell transfection groups. Correlation analyses were performed using linear regression. Diagnostic analyses were performed using ROC curve analysis. In ROC curve analysis, BTN patients or healthy controls were true negative cases, and PTC patients were true positive cases. $P<0.05$ was statistically significant.

\section{Results}

Plasma DLG1-AS1 was upregulated in PTC patients

Levels of DLG1-AS1 in plasma derived from 3 groups of participants (PTC, BTN and Control) were measured and compared by QPCR and ANOVA (one-way) in combination with Tukey test, respectively. Comparing to Control group, significantly higher plasma levels of DLG1-AS1 were found in PTC patients but not in BTN patients (Fig. 1, $p<0.05$ ). 


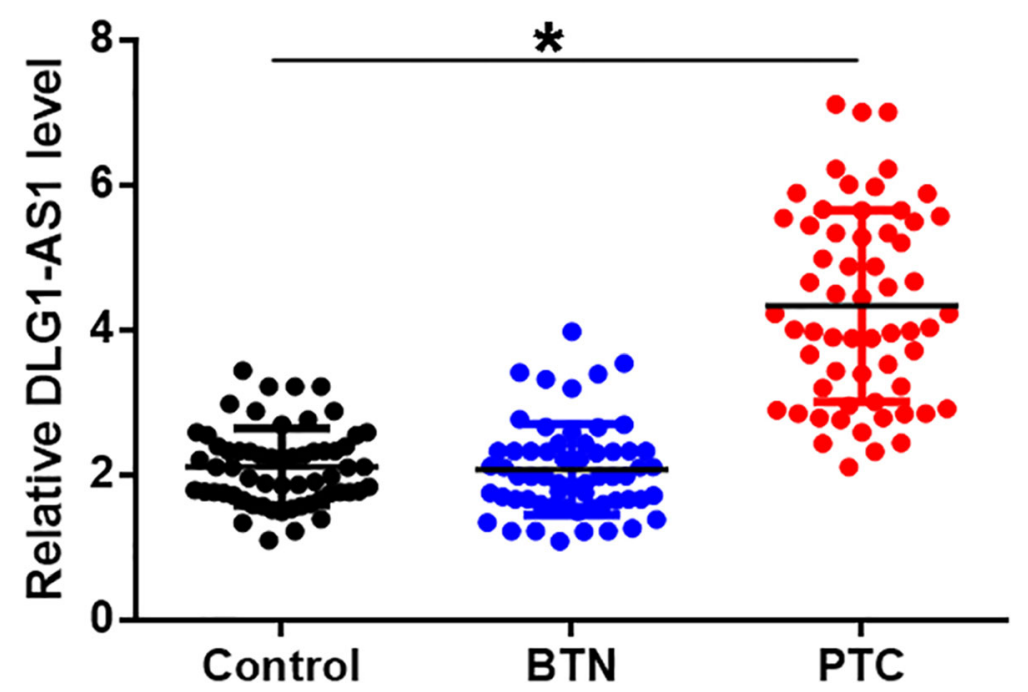

Fig. 1 Plasma DLG1-AS1 was upregulated in PTC patients. Levels of DLG1-AS1 in plasma derived from 3 groups of participants (PTC, BTN and Control) were measured and compared by QPCR and ANOVA (one-way) in combination with Tukey test, respectively. Data were expressed as mean values of 3 replicates, ${ }^{*}, p<0.05$

Plasma DLG1-AS1 had diagnostic potentials for PTC Diagnostic values of DLG1-AS1 for PTC were analyzed using ROC curve through aforementioned methods (Fig. 2). Area under the curve $(\mathrm{AUC})>0.65$ indicates diagnostic potentials. With BTN as true negative cases, AUC was 0.95 (95\% confidence interval: 0.92-0.99; standard error: 0.016). With healthy controls as true negative cases, AUC was 0.96 (95\% confidence interval: 0.93-0.99; standard error: 0.016).

Plasma miR-199a-3p was specifically downregulated in PTC and was positively correlated with DLG1-AS1 in PTC Levels of miR-199a-3p in plasma derived from 3 groups of participants (PTC, BTN and Control) were measured and compared by QPCR and ANOVA (one-way) in combination with Tukey test, respectively. Comparing to Control group, significantly lower plasma levels of miR-199a-3p were found in PTC patients but not in BTN patients (Fig. 3a, $p<0.05$ ). The correlation between miR-199a-3p and DLG1-AS1 was analyzed by linear regression. In PTC patients, plasma levels of DLG1-AS1 were significantly and inversely correlated with the expression levels of miR-199a3p (Fig. 3b). However, the correlation between miR-199a$3 p$ and DLG1-AS1 was not significant in BTN patients and heathy controls (data not shown).

\section{DLG1-AS1 downregulated miR-199a-3p in IHH-4 and MDA-T32 cells}

IHH-4 and MDA-T32 cells were transfected with DLG1-AS1 expression vector or miR-199a-3p mimic. Expression levels of DLG1-AS1 and miR-199a-3p were measured by qPCR. Comparing to $\mathrm{NC}$ and $\mathrm{C}$ two groups, expression levels of DLG1-AS1 and miR-199a$3 p$ were significantly upregulated (Fig. $4 \mathrm{a}, p<0.05$ ). Moreover, miR-199a-3p overexpression failed to significantly affect DLG1-AS1 (Fig. 4b), while DLG1-AS1 overexpression resulted in downregulated miR-199a-3p (Fig. 4c, $p<0.05$ ).
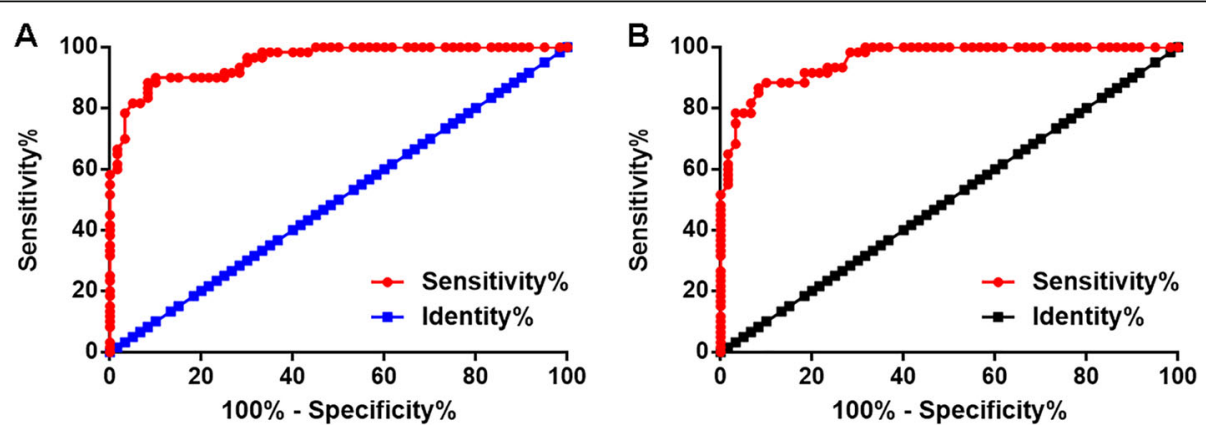

Fig. 2 Plasma DLG1-AS1 had diagnostic potentials for PTC. Diagnostic values of DLG1-AS1 for PTC were analyzed using ROC curve. In ROC curve, BTN patients (a) or healthy controls (b) were true negative cases, and PTC patients were true positive cases 

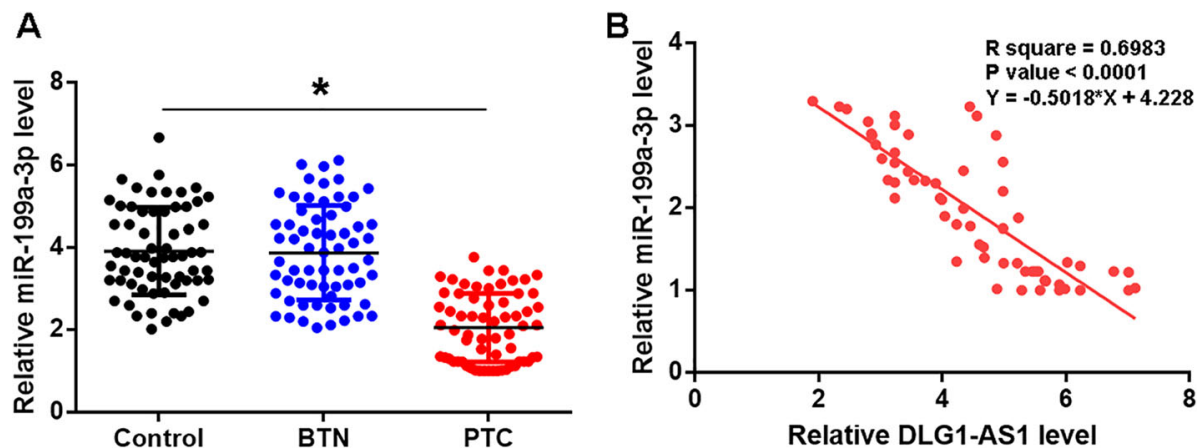

Fig. 3 Plasma miR-199a-3p was specifically downregulated in PTC and was positively correlated with DLG1-AS1 in PTC. Levels of miR-199a-3p in plasma derived from 3 groups of participants (PTC, BTN and Control) were measured and compared by QPCR and ANOVA (one-way) in combination with Tukey test, respectively (a). The correlation between miR-199a-3p and DLG1-AS1 in PTC patients was analyzed by linear regression (b). Data were expressed as mean values of 3 replicates, ${ }^{*}, p<0.05$

DLG1-AS1 promoted PTC cell proliferation through miR199a-3p

The effects of DLG1-AS1 and miR-199a-3p overexpression on the proliferation of $\mathrm{IHH}-4$ (Fig. 5a) and MDAT32 (Fig. 5b) cells were analyzed by CCK-8 assay. Comparing to $\mathrm{C}$ and NC groups, DLG1-AS1 overexpression promoted the proliferation of PTC cells. MiR-199a-3p overexpression played an opposite role and attenuated the effects of DLG1-AS1 overexpression $(p<0.05)$.

\section{Discussion}

This study mainly investigated the roles of DLG1-AS1 in PTC. We found that DLG1-AS1 was upregulated in plasma of PTC patients, and DLG1-AS1 overexpression
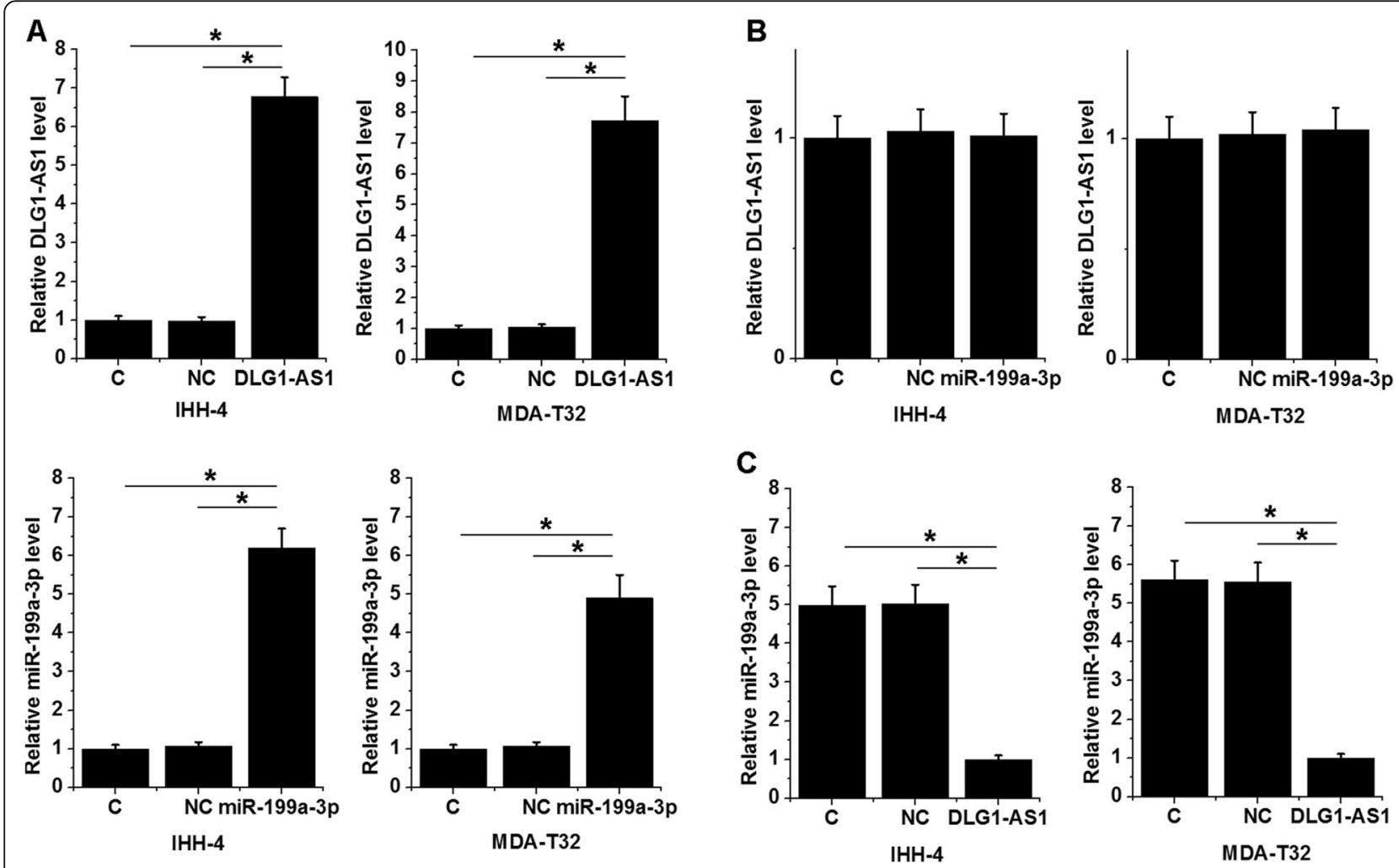

Fig. 4 DLG1-AS1 downregulated miR-199a-3p in IHH-4 and MDA-T32 cells. IHH-4 and MDA-T32 cells were transfected with DLG1-AS1 expression vector or miR-199a-3p mimic. Overexpression of DLG1-AS1 and miR-199a-3p was confirmed by qPCR (a). The effects of miR-199a-3p overexpression on DLG1-AS1 expression (b) and the effects of DLG1-AS1 overexpression on miR-199a-3p expression (c) were also analyzed by qPCR. Data were expressed as mean values of 3 replicates, ${ }^{*}, \mathrm{p}<0.05$ 

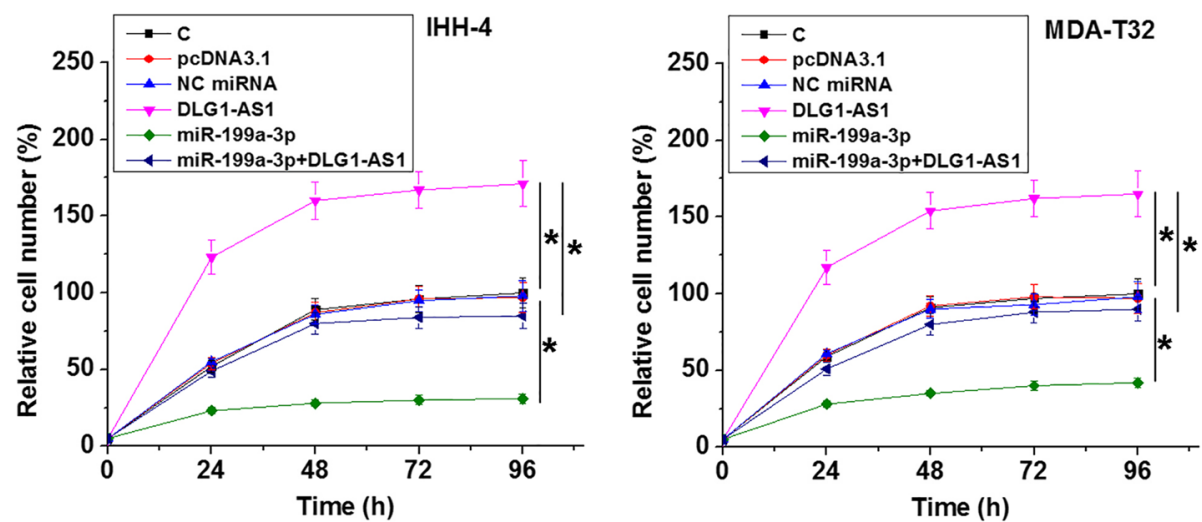

Fig. 5 DLG1-AS1 promoted PTC cell proliferation through miR-199a-3p. The effects of DLG1-AS1 and miR-199a-3p overexpression on the proliferation of IHH-4 (a) and MDA-T32 (b) cells were analyzed by CCK-8 assay. Data were expressed as mean values of 3 replicates, *, $p<0.05$

in PTC cells may downregulate miR-199a-3p to promote the proliferation of PTC cells.

The expression pattern and function of DLG1-AS1 have been investigated in cervical cancer [11]. In cervical cancer, DLG1-AS1 was overexpressed and upregulation of DLG1-AS1 led to the accelerated progression of disease [11]. However, in that study the expression of DLG1-AS1 has only been investigated in tumor and adjacent non-tumor tissues, while the existence of circulating DLG1-AS1 is unknown. In this study we detected the existence of DLG1-AS1 in plasma of all participants. It is known that circulating lncRNAs may serve as systemic signaling to regulate the expression of genes [15]. Therefore, DLG1-AS1 may also regulate gene expression in distant targets after trafficking in blood. Our future studies will explore this possibility.

PTC shares similar imaging features with BTN [16]. Therefore, in clinical practices PTC can be misdiagnosed as BTN, which leads to delayed treatment. Therefore, molecular profiling has been developed to distinguish PTC from BTN [17]. In this study we found that increased plasma levels of DLG1-AS1 can be used to separate early stage PTC patients (stage I and II) from healthy controls and BTN patients. Therefore, measurement of the levels of circulating DLG1-AS1 may assist the early diagnosis of PTC patients, thereby benefitting the survival of PTC patients. We will in future studies include more patients to further analyze the accuracy.

MiR-199a-3p is a well-characterized tumor suppressive miRNA in several types of cancer [12-114]. In hepatocellular carcinoma, miR-199a-3p suppresses cancer cell proliferation by targeting CD44 [12]. In gastric cancer, miR-199a-3p inhibits cancer progression by targeting oncogenic ZHX1 [113]. In a recent study, Minna el at also showed the tumor suppressive role of miR-199a$3 p$ in PTC [14]. In this study we also reported the downregulation of miR-199a-3p in PTC and the increased rate of PTC cell proliferation after miR-199a-3p overexpression. Our data confirmed the tumor suppressive role of miR-199a-3p in PTC. Our study also showed that DLG1-AS1 could downregulate miR-199a$3 p$ to promote cancer cell proliferation. However, the mechanism of the interaction between miR-199a-3p and DLG1-AS1 is unclear. Our future studies will perform deeper investigations.

This study only included Han Chinses, which may provide biased results. The values of DLG1-AS1 in the diagnosis of PTC among other populations remain to be further explored.

\section{Conclusions}

In conclusion, DLG1-AS1 plays oncogenic roles in PTC by downregulating miR-199a-3p to promote cancer cell proliferation. In addition, measurement of the levels of DLG1-AS1 in plasma among the population with high risk of PTC may improve the early diagnosis.

\section{Abbreviations}

> 200 nt, IncRNAs: Long no-coding RNAs; BTN: Benign thyroid nodules; PTC: Papillary thyroid carcinoma

\section{Acknowledgements}

Not applicable.

\section{Authors' contribution}

YFL supervised the whole study, analyzed the data and edited the manuscript.

TH, HW, JMS, JW, FKG, SJL, HW collected and analyzed the data, prepared the figures and manuscript.

All authors have read and approved the manuscript.

\section{Funding}

No funding was obtained for this study.

Availability of data and materials

The analyzed data sets generated during the study are available from the corresponding author on reasonable request. 


\section{Ethics approval and consent to participate}

The present study was approved by the Ethics Committee of Panzhihua Central Hospital. The research has been carried out in accordance with the World Medical Association Declaration of Helsinki. All patients and healthy volunteers provided written informed consent prior to their inclusion within the study.

\section{Consent for publication}

Not applicable.

\section{Competing interests}

The authors declare that they have no competing interests

\section{Author details}

'Department of Nuclear Medicine, Panzhihua Central Hospital, Sichuan Province 617067 Panzhihua City, People's Republic of China. ${ }^{2}$ Department of Ultrasound, Panzhihua Central Hospital, No. 34 YiKang street, Eastern DistrictSichuan Province 617067 Panzhihua City, People's Republic of China.

\section{Received: 2 June 2019 Accepted: 9 October 2019}

\section{0.}

\section{References}

1. Bray F, Ferlay J, Soerjomataram I, et al. Global cancer statistics 2018: GLOBOCAN estimates of incidence and mortality worldwide for 36 cancers in 185 countries. CA Cancer J Clin. 2018;68(6):394-424.

2. Kuo SF, Lin SF, Chao TC, et al. Prognosis of multifocal papillary thyroid carcinoma. Int J Endocrinol. 2013;2013:809382.

3. Pellegriti G, Frasca F, Regalbuto C, et al. Worldwide increasing incidence of thyroid cancer: update on epidemiology and risk factors. J Cancer Epidemiol. 2013;2013:965212.

4. Roh JL, Kim JM, Park Cl. Central lymph node metastasis of unilateral papillary thyroid carcinoma: patterns and factors predictive of nodal metastasis, morbidity, and recurrence. Ann Surg Oncol. 2011;18(8): 2245-50.

5. Randolph GW, Duh QY, Heller KS, et al. The prognostic significance of nodal metastases from papillary thyroid carcinoma can be stratified based on the size and number of metastatic lymph nodes, as well as the presence of extranodal extension. Thyroid. 2012;22(11):1144-52.

6. Sebag F, Vaillant-Lombard J, Berbis J, et al. Shear wave elastography: a new ultrasound imaging mode for the differential diagnosis of benign and malignant thyroid nodules. J Clin Endocrinol Metab. 2010; 95(12):5281-8.

7. Zane M, Agostini M, Enzo MV, et al. Circulating cell-free DNA, SLC5A8 and SLC26A4 hypermethylation, BRAFV600E: a non-invasive tool panel for early detection of thyroid cancer. Biomed Pharmacother. 2013; 67(8):723-30.

8. Fatica A, Bozzoni I. Long non-coding RNAs: new players in cell differentiation and development. Nat Rev Genet. 2014;15(1):7-21.

9. Qiu ZL, Shen $C T$, Sun Z, et al. Circulating long non-coding RNAs act as biomarkers for predicting 1311 uptake and mortality in papillary thyroid cancer patients with lung metastases. Cell Physiol Biochem. 2016;40(6):1377-90

10. Qi P, Zhou X, Du X. Circulating long non-coding RNAs in cancer: current status and future perspectives. Mol Cancer. 2016;15(1):39.

11. Rui $X, X U Y$, Huang $Y$, et al. IncRNA DLG1-AS1 promotes cell proliferation by competitively binding with miR-107 and up-regulating ZHX1 expression in cervical Cancer. Cell Physiol Biochem. 2018;49(5): 1792-803.

12. Henry JC, Park JK, Jiang J, et al. miR-199a-3p targets CD44 and reduces proliferation of CD44 positive hepatocellular carcinoma cell lines. Biochem Biophys Res Commun. 2010;403(1):120-5.

13. Wang Z, Ma X, Cai Q, et al. MiR-199a-3p promotes gastric cancer progression by targeting ZHX1. FEBS Lett. 2014;588(23):4504-12.

14. Minna E, Romeo P, De Cecco L, et al. miR-199a-3p displays tumor suppressor functions in papillary thyroid carcinoma. Oncotarget. 2014; 5(9):2513-28.

15. Anfossi S, Babayan A, Pantel K, et al. Clinical utility of circulating non-coding RNAs_-an update. Nat Rev Clin Oncol. 2018;15(9):541-63.
16. Chan BK, Desser TS, MCDougall IR, et al. Common and uncommon sonographic features of papillary thyroid carcinoma. J Ultrasound Med. 2003;22(10):1083-90

17. Finley DJ, Arora N, Zhu B, et al. Molecular profiling distinguishes papillary carcinoma from benign thyroid nodules. J Clin Endocrinol Metab. 2004; 89(7):3214-23

\section{Publisher's Note}

Springer Nature remains neutral with regard to jurisdictional claims in published maps and institutional affiliations.

\section{Ready to submit your research? Choose BMC and benefit from:}

- fast, convenient online submission

- thorough peer review by experienced researchers in your field

- rapid publication on acceptance

- support for research data, including large and complex data types

- gold Open Access which fosters wider collaboration and increased citations

- maximum visibility for your research: over $100 \mathrm{M}$ website views per year

At $\mathrm{BMC}$, research is always in progress.

Learn more biomedcentral.com/submissions 